\title{
Efectos físicos y emocionales tras la realización de trabajo nocturno continuado en médicos internos residentes
}

\section{Emotional and Phisical Effects After Continued Night Shift by Internal Medical Resident}

\section{Francisco Javier González Espinel', Laura María Blanco Álvarez², Macarena Gálvez Herrer³, María Luisa Rodríguez de la Pinta², María Victoria Casares del Rio', José Manuel de la Torre Robles'}

1. Servicio de Prevención de Riesgos Laborales del Complejo Asistencial Universitario de León. León. España.

2. Servicio de Prevención de Riesgos Laborales del Hospital Universitario Puerta de Hierro Majadahonda. Madrid. España.

3. Tutora docente de la Escuela Nacional de Medicina del Trabajo. Instituto de Salud Carlos III. Madrid. España.

Recibido: 24-10-2019

Aceptado: 09-11-2019

\author{
Correspondencia \\ Francisco Javier Gonzalez Espinel \\ Servicio de Prevención de Riesgos Laborales del Complejo Asistencial Universitario de León. \\ León. España. \\ Correo electrónico: javier.gonzalez.espinel@gmail.com
}

Este trabajo se ha desarrollado dentro del Programa Científico de la Escuela Nacional de Medicina del Trabajo del Instituto de Salud Carlos III en convenio con la Unidad Docente de Medicina del Trabajo de la Comunidad de Madrid y la Unidad Docente de Castilla y León

\section{Resumen}

Introducción: Los Médicos Internos Residentes (MIR) realizan guardias de 17 horas en los hospitales españoles. Dicho trabajo nocturno provoca una alteración del ritmo circadiano que puede desencadenar disfunciones en el estado físico y emocional.

OBJETIVOS: Conocer el grado de afectación física y emocional que generan las guardias en los MIR y los factores que afectan dicha relación.

Material y métodos: Los participantes aleatorizados de un hospital terciario, cumplimentan un cuestionario, antes y después de la realización de la guardia, con variables sociodemográficas y test validados en su versión española: Positive and Negative Affect Schedule, Swedish Occupational Fatigue Inventory y la subescala de apoyo jerárquico de KARASEK.

Resultados: Se realiza análisis estadístico de $n=150$ residentes. De los residentes que conducen tras la guardia, un $17,98 \%$ sufrieron un accidente in itinere. La sensación de bienestar se vio reducida tras la guardia de forma estadísticamente significativa, al contrario que la de malestar $(\mathrm{p}<0,05)$. Y el mayor grado de fatiga tras la guardia se centra en la somnolencia y en menor grado la falta de motivación.

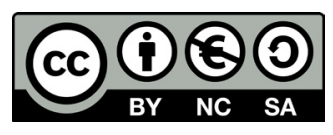

https://creativecommons.org/licenses/by-nc-sa/4.0/

Efectos físicos y emocionales tras la realización de trabajo nocturno continuado en médicos internos residentes Francisco Javier González Espinel, Laura María Blanco Álvarez, Macarena Gálvez Herrer, María Luisa Rodríguez de la Pinta, 
Conclusiones: Este estudio supone una estimación validada de los efectos del trabajo nocturno continuado de los MIR. Los resultados obtenidos podrían sugerir replantearse la organización del trabajo debido a los efectos negativos para el bienestar de los médicos del futuro.

Med Segur Trab (Internet). 2019;65(257):252-260

Palabras clave: Médicos, trabajo nocturno, ritmo circadiano, efectos en la salud

\section{Abstract}

Introduction: Medical Internal Residents perform 17-hour shifts at Spanish hospitals. Such night work causes an alteration of the circadian rhythm that can trigger disturbances in the physical and emotional state.

Objectives: To know the degree of physical and emotional affectation that the night shifts generate in Medical Residents and the factors that affect this relationship.

Material and methods: Before and after their 17-hour shift, the randomized participants from a tertiary referral hospital complete a questionnaire with socio-demographic variables and tests validated in their Spanish version: Positive and Negative Affect Schedule, Swedish Occupational Fatigue Inventory and the subscale of hierarchical support of KARASEK

Results: Statistical analysis of $n=150$ residents of a hospital was carried out. Among the residents who drive after the 17-hour shift, $17.98 \%$ suffered a commuting accident. The pleasant experiences were reduced after shifts in a statistically significant difference $(p<0.05)$, compared to the discomforting ones. The highest degree of fatigue after shifts is focused on drowsiness, and to a lesser degree, on a lack of motivation.

Conclusions: This study supposes a validated estimate of the effects of the continuous work shift of Medical Residents. The results obtained could suggest reconsidering the organization of work given the negative effects for the well-being of future physicians.

Med Segur Trab (Internet). 2019;65(257):252-260

Keywords: Physicians, shift work, circadian rhythm, health impacts 


\section{INTRODUCCIÓN}

Los Médicos Internos Residentes (MIR) realizan su formación médica durante 4 o 5 años en los hospitales españoles para la obtención de las distintas especialidades, constituyendo una etapa profesional en la que se deben consolidar amplias competencias. Cada año, se incorporan de media 6000 nuevos profesionales médicos a la formación vía MIR, constituyendo un gran volumen laboral que dará lugar a los médicos del mañana.

A lo largo de este periodo, los residentes se incorporan al sistema sanitario asumiendo paulatinamente responsabilidades profesionales en entornos laborales complejos ${ }^{1}$.

La mayoría de los residentes tienen programado en su plan formativo la realización de guardias en el servicio de urgencias durante el primer año, siendo esta, la primera toma de contacto con la presión asistencial, falta de apoyo, dilemas éticos o contacto con el dolor y la muerte de los pacientes $^{2}$. Este ambiente formativo y laboral conforma una etapa estresante que conduce al residente a una situación de especial vulnerabilidad psicológica. Numerosos estudios han demostrado la existencia de diferencias de género en cuanto a la salud mental y los estados de depresión y ansiedad. En este sentido, es frecuente encontrar en las mujeres resultados más desfavorables a nivel psicológico. Este hecho es de suma relevancia si se tiene en cuenta que, en los últimos años, la profesión médica está sufriendo una gran feminización y se espera que el porcentaje de mujeres médico siga en aumento ${ }^{3}$.

Los turnos de guardias en la mayoría de los hospitales españoles se dividen en dos modalidades: turnos de 17 horas y de 24 horas. Los primeros comienzan a las 15 horas y finalizan a las 8 horas de la mañana del día siguiente. Estos turnos de guardias se realizan en días laborales, por lo que el profesional ya lleva trabajando 8 horas en el turno de mañana. Por otra parte, los segundos, comienzan a las 9 horas de la mañana y finalizan a la misma hora del día siguiente, éstos últimos se suelen realizar los fines de semana. En cualquiera de los dos tipos, existe un componente de horario nocturno asociado. A esto se añade el hecho de que los profesionales de determinados servicios médicos y quirúrgicos tengan que trabajar el turno siguiente a la guardia por las necesidades de estos servicios, llevando un total de 32 horas seguidas trabajando. Este hecho constatable en la práctica médica diaria es contrario al artículo 5 del Real Decreto 1146/2006, de 6 de octubre por el que se establece un descanso continuo de 12 horas tras 24 horas de trabajo ininterrumpido ${ }^{4}$.

Recientemente con la aprobación del Real Decreto-ley 8/2019, de 8 de marzo, de medidas urgentes de protección social y de lucha contra la precariedad laboral en la jornada de trabajo, los MIR tendrán la posibilidad de registrar y justificar las horas extras realizadas como personal laboral hospitalario. Este hecho, puede suponer un cambio en la dinámica de los descansos de los MIR, que sólo el tiempo podrá decir si repercute positivamente en su salud'5

El trabajo nocturno que realizan los médicos internos residentes durante las guardias provoca alteración del ritmo circadiano, la modificación de las horas de sueño y la alteración en el ciclo luz-oscuridad. Esto se asocia a enfermedades crónicas como diabetes mellitus, hipercolesterolemia, trastornos digestivos y cardiovasculares, constituyendo un factor de riesgo para trastornos eventualmente mortales ${ }^{6,7}$. También la pérdida de sueño puede desencadenar ansiedad, depresión y burnout, que como indican diversos estudios conlleva a un incremento de la automedicación principalmente durante el primer año de residencia, así como una disminución de la calidad de vida extralaboral ${ }^{8,1}$.

Otro tema de vital importancia para el mundo médico son los accidentes laborales, que se incrementan por la privación del sueño, con descenso del nivel de alerta e incremento del tiempo de reacción. Se ha estudiado el efecto de los turnos nocturnos de larga duración, demostrándose que aumentan los errores médicos en tareas clínicas como la prescripción farmacológica 9,10 .

Efectos físicos y emocionales tras la realización de trabajo nocturno continuado en médicos internos residentes Francisco Javier González Espinel, Laura María Blanco Álvarez, Macarena Gálvez Herrer, María Luisa Rodríguez de la Pinta, María Victoria Casares del Rio, José Manuel de la Torre Robles 
En 1984 en el New York Hospital, tras el fallecimiento de una adolescente de 18 años por un supuesto error médico por incompatibilidad de fármacos, se instauró la Ley Libby Zion que limita a 80 horas de trabajo máximo a la semana a los médicos internos residentes de Nueva York ${ }^{11}$.

No es menos preocupante el hecho de que se produzcan accidentes y errores médicos sino también la ausencia de regulación que ayude a garantizar la seguridad pública que otras industrias, como la nuclear y la aeronáutica, ya poseen ${ }^{11,12}$. Porque no sólo se trata de proteger al público sino también a los médicos internos residentes de posibles daños prevenibles. En base a esta idea, Estados Unidos publicó un informe en el que se especifican recomendaciones sobre nuevos horarios de 12 o 16 horas por turno y un mínimo de 10 horas de descanso entre turnos. Así mismo, se incentiva el aumento de la supervisión, principalmente en unidades de pacientes críticos y se valora la seguridad, siendo el hospital el que proporcione transporte a los médicos residentes que refieran sentirse demasiado cansados para conducir, disminuyendo el riesgo de los accidentes in itinere $^{12}$. Según estudios, los residentes tienen un menor rendimiento en la conducción, se ve aumentado el tiempo de reacción, tienen mayor dificultad para controlar la velocidad y más déficits de atención cuando se compara su trabajo en turnos diurnos versus nocturnos $^{13,9,10}$.

Según el BOE, todos los adjudicatarios de plaza por el sistema de residencia se someterán a examen médico por el servicio de Prevención de Riesgos Laborales para comprobar que no padecen enfermedad ni están afectados por limitación física, psíquica o sensorial que sea incompatible con las actividades profesionales que el correspondiente programa formativo oficial exija al residente ${ }^{14}$. Sin embargo, no se hace obligatorio un examen médico posterior que valore los posibles cambios en su salud que el ejercicio de su profesión ocasionara.

La falta de estudios previos sobre los efectos a corto plazo de las guardias en la salud de los MIR, nos obliga a intentar arrojar luz en este tema tan frecuente y relevante.

\section{OBJETIVOS}

El objetivo principal de este trabajo, por tanto, se centra en la evaluación de los efectos a corto plazo en la salud física y emocional de los MIR que realizan guardias e identificar qué factores y en qué medida afectan a esta relación.

Entre los objetivos específicos de este estudio destacamos los siguientes:

- Estudiar la posible asociación entre el año de residencia y el número medio de horas dormidas en cada guardia.

- Analizar la relación entre el grado de supervisión subjetiva y el tipo de especialidad.

- Calcular el porcentaje de residentes que realiza libranza de guardia por tipo de especialidad médica o quirúrgica, así como el número de horas extra que trabaja en el hospital al día siguiente.

- Evaluar si los sentimientos positivos y negativos se ven afectados por igual tras la realización de la guardia.

- Ordenar de mayor a menor frecuencia de aparición, cada uno de los 5 subgrupos de fatiga laboral del cuestionario Swedish Occupational Fatigue Inventory: falta de energía, esfuerzo físico, disconfort físico, falta de motivación y somnolencia.

- Estimar qué porcentaje de los MIR consumen analgésicos para sobrellevar los efectos de una guardia y su relación con el género.

- Estimar el porcentaje de residentes que sufren un accidente-incidente in itinere en la mañana siguiente a la guardia, así como su relación con el tipo de especialidad médica o quirúrgica.

Efectos físicos y emocionales tras la realización de trabajo nocturno continuado en médicos internos residentes 


\section{MATERIAL Y MÉTODOS}

\section{Diseño y población del estudio}

El diseño del presente estudio consta de una parte descriptiva y otra cuasiexperimental (antes y después). La población de estudio son los médicos internos residentes de un hospital de tercer nivel, que realizan guardias durante la residencia.

\section{Método estadístico}

De la plantilla de los médicos internos residentes de dicho hospital se seleccionaron un total de 150 participantes por muestreo aleatorio, estratificado por año de residencia, y con reposición de elementos.

En la primera aleatorización, se consigue la participación de 142 residentes, por lo que es necesaria la reposición con 8 sujetos del mismo cluster al cual pertenece el sujeto que rechaza su participación o no cumple los criterios necesarios (lost to follow-up).

De los 8 sujetos, se consigue la participación de 6 , por lo que se procede nuevamente a la reposición de 2 sujetos.

\section{Control de sesgos y normas éticas}

El rechazo, aunque escaso, en la participación en este estudio es una pérdida no informativa (lost to follow-up) por lo que desconocemos si pudiera existir un pequeño sesgo de selección en el cual los sujetos más cansados rechacen su participación.

En cuanto a la exposición de cada sujeto, los investigadores no modifican la exposición a las variables independientes, siendo cada sujeto su propia referencia antes y después de la realización de la guardia.

Por último, los sujetos participantes no reciben información acerca de las hipótesis del estudio para que su participación y/o respuestas no dependan de la misma.

Previo al desarrollo del proyecto de investigación, se expuso el diseño y metodología al comité ético del hospital el cuál autorizó su realización.

\section{Recogida de datos}

La recogida de los datos se realiza mediante la utilización de cuestionarios anónimos y codificados en formato papel, para ser cumplimentados antes y después de la guardia por cada uno de los participantes. El cuestionario se cumplimenta con la autorización expresa por escrito de cada uno de los participantes. Los resultados son anónimos y codificados, asegurando la privacidad de los mismos. Estos datos sólo son accesibles a los dos investigadores principales, y en ningún caso se verán usados para perjuicio de la muestra a estudio.

En relación al contenido de los cuestionarios utilizados para recoger la información que se desea estudiar, este consta de una parte inicial de variables sociodemográficas de diseño ad-hoc que evalúa las variables tanto sociodemográficas, como laborales y de seguridad vial. Además, se utilizan tres escalas validadas: la escala SOFI, que es la versión española del Swedish Occupational Fatigue Inventory (SOFI) de González y cols., 2005 utilizada para la evaluación de la salud física a través de una medida de fatiga y somnolencia; la escala PANAS, que es la versión española del Positive and Negative Affect Schedule, de Sandín y cols., 1999, utilizada para la evaluación de los estados afectivos positivos y negativos y por último la escala KARASEK modificada en la que se incluyen los ítems de la dimensión de "Apoyo por parte de los supervisores" del Job Content Questionnaire (versión 1.5) de Karasek y cols. 1993, utilizada para la evaluación de la percepción de estilos de supervisión por el mando directo.

Efectos físicos y emocionales tras la realización de trabajo nocturno continuado en médicos internos residentes Francisco Javier González Espinel, Laura María Blanco Álvarez, Macarena Gálvez Herrer, María Luisa Rodríguez de la Pinta, 
Las escalas de SOFI y PANAS se cumplimentan en dos momentos distintos, es decir, antes y después de la realización de la guardia por el médico interno residente, mientras que la escala Karasek sólo se cumplimenta tras la finalización de dicha guardia.

\section{Criterios de inclusión y exclusión}

Entre los criterios de inclusión se encuentran los MIR que estén realizando guardias durante el período de recogida de datos de tres meses de duración y los MIR pertenecientes al propio hospital a estudio.

Entre los criterios de exclusión se encuentran los MIR exentos de la realización de guardias durante el período de estudio, los MIR que realizan guardias inferiores a 17 horas de duración, los MIR rotantes o cuya plaza adjudicada no se encuentre en el hospital a estudio, los MIR que hayan realizado una guardia en las últimas 48 horas antes de la contestación del cuestionario y los cuestionarios no cumplimentados al inicio y finalización de la guardia.

\section{Variables a estudio}

Con respecto a la clasificación de las variables estudiadas, algunas de ellas hacen referencia a aspectos sociodemográficos como el género o el tipo de especialidad. En referencia a las variables relacionadas con las guardias, se estudia el número de guardias realizadas al mes de media en cada uno los servicios médicos o quirúrgicos y el grado de supervisión.

Respecto a las variables que hacen referencia a las consecuencias directas de una guardia destacamos: el haber sufrido un incidente/accidente in itinere tras la realización de una guardia, el consumo de medicación analgésica, el grado de fatiga física (Swedish Occupational Fatigue Inventory, SOFI) y el grado de afectación emocional tras la guardia diferenciando entre con sentimientos positivos y negativos (Positive and Negative Affect Schedule, PANAS)

\section{Análisis de los datos}

El análisis estadístico se realiza mediante el programa estadístico SPSS Statistics v24 en función del tipo de variable. En este estudio contamos con indicadores cualitativos dicotómicos como la aparición o no de un hecho (como un accidente in itinere) o la pertenencia a una especialidad médica o quirúrgica. Contamos también con indicadores cuantitativos discretos con una puntuación total recogida a través del sumatorio de escalas tipo Likert (SOFI, PANAS y KARASEK).

\section{RESULTADOS}

1. Variables Sociodemográficas de la muestra.

- La muestra estudiada ( $n=150)$ está conformada por un $43.67 \%$ de hombres y un $56.33 \%$ de mujeres.

- Respecto a la edad de la muestra estudiada, el $14.2 \%$ es $\leq 25$ años, el $71.6 \%$ entre 26-30 años, el 11.3\% entre 31-36 años y el 2.9\% > 36 años.

2. Guardias

- El número medio de guardias al mes por año de residencia fue: R1 (3,56 guardias), R2 (3,66 guardias), R3 (4,91 guardias), R4 (5,2 guardias), R5 (5,53 guardias). Observándose que la media de horas dormidas en cada guardia aumenta con cada año de residencia.

- Los porcentajes de libranza por tipo de especialidad fueron (Ver Figura 1)

Efectos físicos y emocionales tras la realización de trabajo nocturno continuado en médicos internos residentes 
Figura 1. Porcentajes de libranza postguardia por tipo de especialidad.

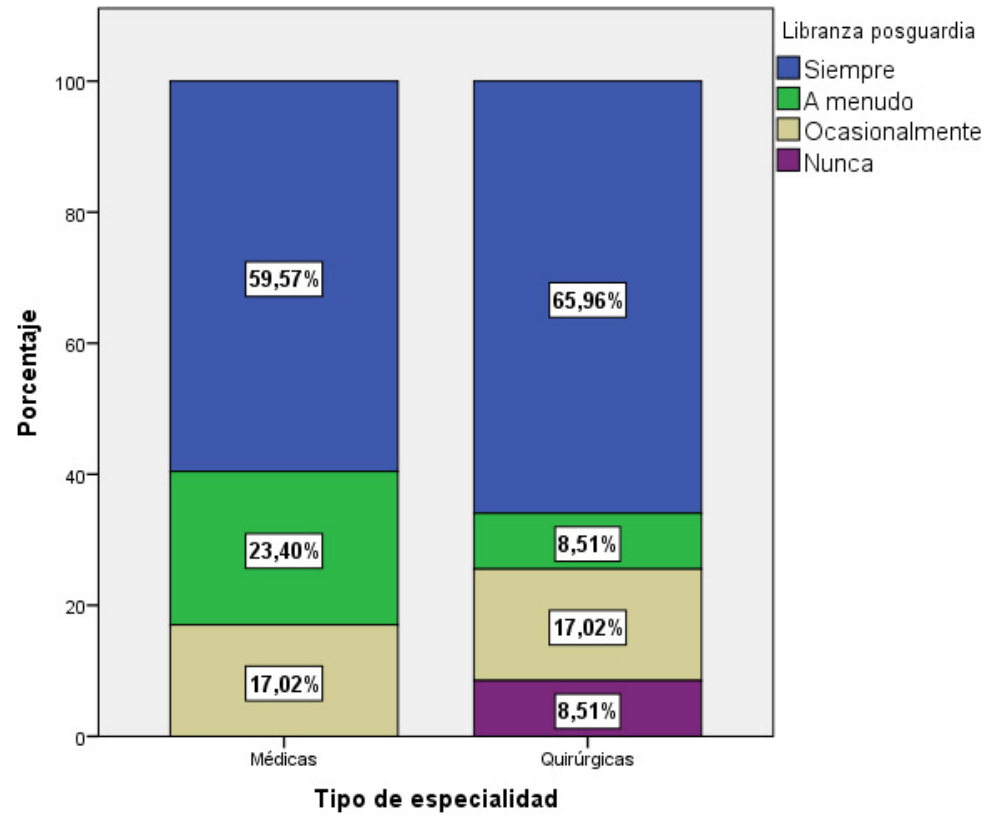

- El número medio de horas extras por tipo de especialidad entre los sujetos que no libran las guardias fue: Médicas 2,65 horas/guardia y quirúrgicas 2,51 horas/guardia.

- El número medio de horas extras estratificado por año de residencia entre los sujetos que no libran las guardias fue: R1 (1,83 horas), R2 (1,67 horas), R3 (3,15 horas), R4 (3,71 horas), R5 (3,3 horas).

- El apoyo jerárquico percibido por año de residencia empleando el test KARASEK fue (Ver figura 2).

Figura 2. Apoyo jerárquico percibido por año de residencia.

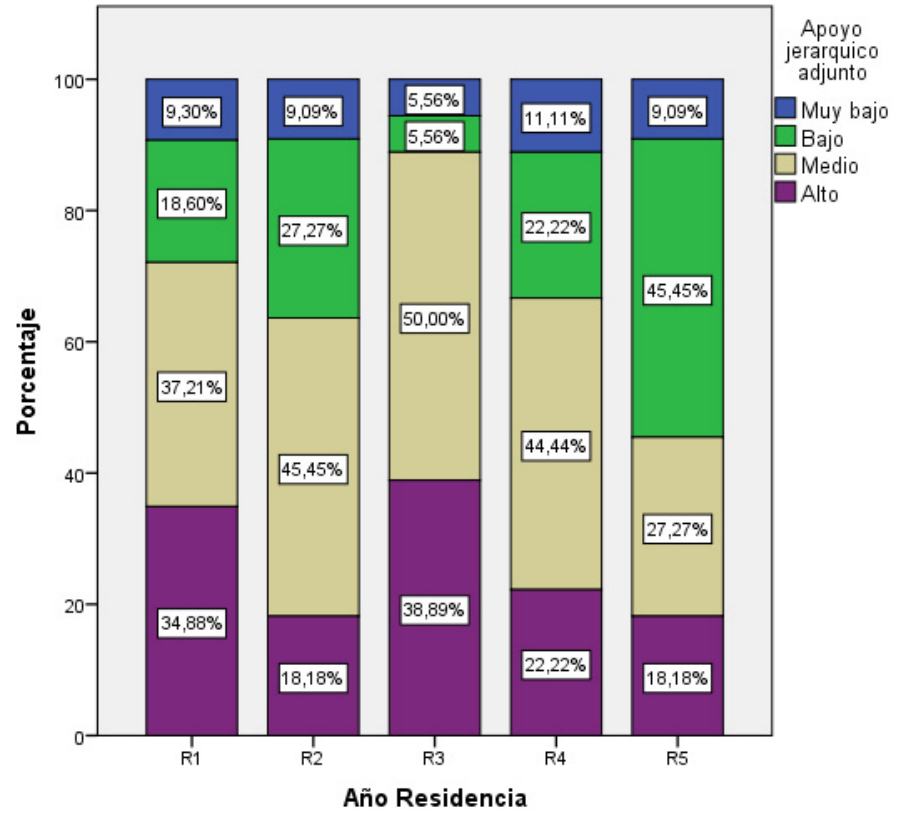

3. Guardias y medicación.

- Medicación: Debido al reducido porcentaje de personas que han necesitado medicación para dormir tras la guardia (2.8\%), no se puede establecer ningún 
tipo de asociación por género. Sin embargo, respecto al consumo de medicación analgésica y género, el test de Chi Cuadrado de Pearson $\chi^{2}$ arroja un valor de 5,4 lo cual es significativo $(<0,05)$ para un grado de libertad entre la mayor asociación positiva de hombre y consumo de analgésicos para paliar los efectos de la guardia en comparación con las mujeres estudiadas.

4. Conducción.

- El 63,1\% de los residentes, conducen a la mañana siguiente a la guardia, de los cuales el $52.81 \%$ ha sentido quedarse dormido.

En este aspecto, establecemos la hipótesis nula de que la proporción de residentes que han sentido quedarse dormidos al volante es igual para residentes quirúrgicos y médicos y cualquier diferencia observada se debería simplemente al azar. En su análisis el test de Chi-cuadrado de Pearson $\chi^{2}$ arroja un valor de 8.72 para un grado de libertad por lo que la probabilidad de encontrar un resultado tan alejado o más de nuestra hipótesis nula es < 0.05 (error alfa de 0,05). Por lo tanto, rechazamos la hipótesis nula de igualdad, deduciendo la asociación de que los residentes de especialidades quirúrgicas se han sentido más dormidos al volante que los de especialidades médicas.

- De los residentes que conducen a la mañana siguiente a la guardia, el $17.98 \%$ ha sufrido un incidente/accidente in itinere.

5. Efectos en la fatiga física.

Test: Swedish Occupational Fatigue Inventory.

- La medición se realizó con la media de las variaciones (antes-después de la guardia) en cada una de las subescalas. La variación media de mayor a menor repercusión fue: $1^{\circ}$ Somnolencia $\left(14,58 \pm 0,66\right.$ puntos), $2^{\circ}$ Falta de energía $(13,09 \pm 0,62$ puntos $), 3^{\circ}$ Falta de motivación $(10,14 \pm 0,52$ puntos $), 4^{\circ}$ Disconfort físico $(5,56 \pm 0,61$ puntos $) 5^{\circ}$ Esfuerzo físico $(2,32 \pm 0,53$ puntos $)$

6. Efectos emocionales (Sentimientos positivos y negativos)

Test: Positive and Negative Affect Schedule.

- Sentimientos positivos (entusiasmo, decisión, alerta ): La media obtenida antes de la realización de la guardia fue de 29,29 \pm 0.62 y después de 21,52 \pm 0.67.

- Sentimientos negativos (vergüenza, inseguridad, nerviosismo ): La medida obtenida antes de la realización de la guardia fue de 20,65 \pm 0,66 y después $20,53 \pm 0.67$.

El análisis estadístico mediante el test de Wilcoxon, arrojó un resultado estadísticamente significativo $(<0,05)$ mostrando una mayor reducción de los sentimientos positivos que negativos tras la realización de la guardia.

\section{CONCLUSIONES}

Este estudio, supone una estimación validada de la diversidad de efectos que tiene el trabajo nocturno continuado en los MIR. Por tanto, constata los efectos físicos, emocionales, accidentabilidad in itinere y su grado de asociación con diversos factores.

Se trata de un estudio con un tamaño muestral reducido en comparación con el total de residentes en España. Sin embargo, su aplicación y reproducción en distintos hospitales pudiera poner sobre aviso a los Servicios de Prevención de Riesgos Laborales respecto a los profesionales más vulnerables.

Cabe destacar el elevado porcentaje de residentes conductores que han sentido quedarse dormidos al volante $(52,81 \%)$, lo cual es concordante con que el principal efecto físico referido tras la guardia sea la somnolencia. Este hecho, pone de manifiesto la

Efectos físicos y emocionales tras la realización de trabajo nocturno continuado en médicos internos residentes 
importancia que puede tener para los Servicios de Prevención, el formar a los residentes sobre algo tan poco investigado como es el accidente in itinere y las graves consecuencias que pudieran derivarse para ellos y terceras personas.

Por otro lado, la mayor afectación en los sentimientos positivos (entusiasmo, decidido, alerta...) en relación a los negativos (avergonzado, inseguro, nervioso...) tras la guardia habla a favor de una influencia menos dañina emocionalmente de las guardias para el MIR. De esta forma, pudiera resultar interesante el estudio de la relación entre los residentes con mayor afectación de sentimientos negativos como factor predictor en el desarrollo futuro de Burnout.

Por todo ello, se constata la gran variedad de efectos que tiene el trabajo nocturno continuado en los MIR y la posibilidad de emplear herramientas para su cuantificación. De este modo, podremos implementar acciones en favor de la seguridad, bienestar físico y mental de los médicos del mañana.

\section{REFERENCIAS BIBLIOGRÁFICAS}

1. Blancafort BS. Estudio sobre la salud de los residentes de Cataluña [Internet]. Fundación Galatea; 2009. (Informes y estudios). Disponible en: https://books.google.es/books?id=h0mvnAEACAAJ

2. Martínez OF, Cabrera CH, Tapia SM, Suárez SM, García BG del R. Burnout en médicos residentes que realizan guardias en un servicio de urgencias. Emergencias: Revista de la Sociedad Española de Medicina de Urgencias y Emergencias. 2007;19(3):116-21.

3. Fernández-Prada M, González-Cabrera J, Torres G F, Iribar-Ibabe C, María Peinado J. [Gender influence on health related quality of life among resident physicians working in an emergency department]. Rev Med Chil. febrero de 2014;142(2):193-8.

4. Real Decreto 1146/2006, de 6 de octubre,por el que se regula la relación laboral especial de residencia para la formación de especialistas en Ciencias de la Salud. (Boletín Oficial del Estado, número 240 de 07 de octubre de 2006)

5. Real Decreto-ley $8 / 2019$, de 8 de marzo, de medidas urgentes de protección social y de lucha contra la precariedad laboral en la jornada de trabajo.

6. Madrigal B, Imelda A, Mora V, José E, Acosta S, José F, et al. El trabajo nocturno y sus implicaciones en la salud de médicos, enfermeras y oficiales de seguridad de los hospitales de la Caja Costarricense del Seguro Social en Costa Rica. Medicina Legal de Costa Rica. marzo de 2013;30(1):17-36.

7. Tobaldini E, Cogliati C, Fiorelli EM, Nunziata V, Wu MA, Prado M, et al. One night on-call: sleep deprivation affects cardiac autonomic control and inflammation in physicians. Eur J Intern Med. octubre de 2013;24(7):664-70

8. Kassam A, Horton J, Shoimer I, Patten S. Predictors of Well-Being in Resident Physicians: A Descriptive and Psychometric Study. J Grad Med Educ. marzo de 2015;7(1):70-4.

9. Rosenbluth G, Landrigan CP. Sleep science, schedules, and safety in hospitals: challenges and solutions for pediatric providers. Pediatr Clin North Am. diciembre de 2012;59(6):1317-28.

10. Anderson C, Sullivan JP, Flynn-Evans EE, Cade BE, Czeisler CA, Lockley SW. Deterioration of neurobehavioral performance in resident physicians during repeated exposure to extended duration work shifts. Sleep. 1 de agosto de 2012;35(8):1137-46.

11. Kramer M. Sleep Loss in Resident Physicians: The Cause of Medical Errors? Front Neurol [Internet]. 20 de octubre de 2010 [citado 7 de julio de 2017];1. Disponible en: http://www.ncbi.nlm.nih.gov/pmc/articles/ PMC3008836/

12. Blum AB, Shea S, Czeisler CA, Landrigan CP, Leape L. Implementing the 2009 Institute of Medicine recommendations on resident physician work hours, supervision, and safety. Nat Sci Sleep. 2011;3:47-85.

13. Huffmyer JL, Moncrief M, Tashjian JA, Kleiman AM, Scalzo DC, Cox DJ, et al. Driving Performance of Residents after Six Consecutive Overnight Work Shifts. Anesthesiology. Junio de 2016;124(6):1396-403.

14. Real Decreto 1146/2006, de 7 de octubre, por el que se regula la relación laboral especial de residencia para la formación de especialistas en Ciencias de la Salud. (Boletín Oficial del Estado, número 240, de 7 de octubre de 2006). 